\title{
Determining the Sign of the Index in Metamaterials Composed of Split Ring Resonators and Strip Wires Using Dispersion Diagrams or the Insertion Phase
}

\author{
J. F. Woodley, M. S. Wheeler, M. Mojahedi \\ Electromagnetics Group, Edward S. Rogers Sr. Department of Electrical and Computer \\ Engineering, University of Toronto, Toronto M5S 2E4, Canada
}

\begin{abstract}
Using full wave simulations the behavior of two structures composed of split ring resonators (SRRs) and strip wires (SWs) is examined. In the region where the real parts of the permittivity and permeability are both expected to be negative both structures exhibit a transmission peak, a property which is generally assumed to imply a negative index of refraction. However, through an analysis of the dispersion characteristics and insertion phase of the two structures it is shown that only the index in the first structure, in which the SRRs and SWs are printed on opposite sides of a dielectric substrate, is negative in the passband. In the second structure, in which SRRs and SWs are printed on the same side of the substrate, the index in the passband is positive. Therefore the emergence of transmission peaks does not provide sufficient evidence of the existence of a negative index of refraction. To determine the correct sign of the index two methods are investigated. The first uses the transmission phase for propagation through various lengths of the structure and the second utilizes its dispersion diagrams. The dependence of the sign of the index on the dimensions of the unit cell size is also examined.
\end{abstract}

\section{INTRODUCTION}

The idea of a negative index of refraction was first introduced in 1967 by Veselago when he posed that such behavior would require simultaneous negative real parts of the electric permittivity and magnetic permeability [1]. In his paper, he dubbed a medium with these properties a left-handed medium (LHM) because of the left-handed relationship between the electric field, the magnetic field, and the propagation vector $\vec{k}$. Veselago's idea of a negative index medium, however, could not be corroborated experimentally at the time due to a lack of suitable media with the required negative parameters.

In 1996 a negative permittivity structure was developed which exhibits a plasma frequency in the microwave regime [2]. This structure consists of an array of strip wires (SW) and can produce the desired behavior without suffering relatively large losses because of its low plasma frequency. Later, in 1999 a negative permeability medium was constructed using an array of split ring resonators (SRRs) [3]. By combining these two structures the first negative index medium was developed and it was shown that in the region where both the real parts of the electric permittivity and magnetic permeability were expected to be negative a transmission passband (i.e. a peak) appeared, a result that is generally taken to imply the existence of the negative index of refraction [4]. The negative index behavior of this medium was later corroborated by an experiment in which a wave was shown to refract negatively at the interface between this structure and air [5]. This work led to an eruption of papers on the topic of LHMs [6-10]. In general, the transmission magnitude for propagation through a medium is used to determine whether the medium is LHM or not. In other words, if in the region where the real parts of the electric permittivity and magnetic permeability are assumed to be negative a transmission peak is observed then the structure is assumed to exhibit LHM behavior [4, 7, 11]. This emergence of a transmission peak, however, is not sufficient evidence that the structure exhibits left handedness. If we consider the SRR and SW structures each produces the required negative behavior when they are isolated from each other, however, their individual field patterns interfere when they are combined into a single structure. This interference can be significant enough to weaken or even destroy the LHM behavior, although a transmission peak and a passband can still be observed. It is therefore desirable to use other "diagnostic tools" such as calculating or measuring the insertion phase and/or dispersion diagrams when determining whether the structure exhibits true negative index behavior. 
This paper is organized as follows. The transmission magnitude through two composite media composed of SRRs and SWs is examined in section II. In section III the handedness of these structures is determined by considering the incurred phase for propagation through them. The dispersion diagrams of these structures are then investigated in section IV. The effect of the unit cell size on the sign of the index in one of the structures is further investigated in section V. Section VI provides our final thoughts and conclusions.

\section{TRANSMISSION MAGNITUDE}

Two structures composed of SRRs and SWs printed on a dielectric substrate will be considered in this paper. In both structures the ring dimensions are identical and are given in Fig. 1. The SWs were also identical for both structures. In the first structure the rings and strips are printed on opposite sides of the substrate [Fig. 2(a)] and in the second structure the rings and strips are printed on the same side of the substrate [Fig. 2(b)]. We will refer to these as the opposite side (OS) and same side (SS) structures respectively.

\section{Insert Fig. 1 \\ Insert Fig. 2}

The transmission behavior of similar structures has been investigated both through simulations and experimental measurements by other groups $[7,12,13]$. In the previous work, when a transmission peak was observed at frequencies where the permittivity and permeability were expected to be negative it was concluded that this was indicative of a negative index of refraction. As we shall see however, this emergence of a passband and a transmission peak does not supply sufficient evidence that LHM behavior exists.

Using Ansoft HFSS, a commercial full wave finite element simulation package, the transmission through the two SRR and SW configurations was simulated. Figure 2 shows the unit cells of the simulated structures. In each case the $z$-faces of the unit cell were assigned the characteristics of a perfect electric conductor (PEC) forcing the electric field to be polarized parallel to the SWs and exciting their negative permittivity behavior. The $x$-faces were given the characteristics of a perfect magnetic conductor (PMC) ensuring that the negative permeability behavior of the rings was excited. In the $y$-direction the unit cells were repeated 4 times so that transmission through a 4 unit cell structure could be simulated. The unit cell in the SS case had dimensions $4 \times 2.5 \times 2.5 \mathrm{~mm}$ while the unit cell in the OS case had dimensions $2.5 \times 2.5 \times 2.5 \mathrm{~mm}$ (the reason for the difference in the $x$-dimension of the two unit cells will be discussed in sections III and VI). Figure 3 shows the simulated transmission magnitude for the two structures where solid vertical lines (dashed) indicate the regions of transition from RHM (LHM) to LHM (RHM) behavior.

\section{Insert Fig. 3}

In both cases there is a clear peak in the transmission magnitude (at $22.6 \mathrm{GHz}$ for the OS case and $22.75 \mathrm{GHz}$ for the SS case). These peaks occur in the regions where the real parts of the electric permittivity and magnetic permeability are expected to be negative. Therefore, it is tempting to conclude that both of the structures being considered exhibit negative index behavior. Before making such conclusions however, let us perform more thorough investigations on these structures and examine their transmission phases and dispersion relations.

\section{TRANSMISSION PHASE}

For a medium with phase index $n(\omega)$ the transmission phase for propagation through a length $L$ of the structure is given by

$$
\phi=-n(\omega) L \frac{\omega}{c}
$$

where $c$ is the speed of light in vacuum. For propagation through different lengths $L_{1}$ and $L_{2}$ of this medium the difference in the transmission phase is 


$$
\Delta \phi=-n(\omega)\left(L_{2}-L_{1}\right) \frac{\omega}{c}
$$

Using (2) the sign of the effective index in a structure can be determined. If we assume $L_{2}>L_{1}$ then in an RHM the difference $\Delta \phi$ will be negative since $n(\omega)>0$. In an LHM, on the other hand, since $n(\omega)<0$ the difference $\Delta \phi$ will be positive. Another way to put this is that in an RHM the insertion phase of the longer structure will lie below that of the shorter structure, whereas the opposite is true for an LHM. Figure 4 shows the transmission phase for propagation through 1, 2, 3, and 4 unit cells of the SRR and SW configurations ${ }^{1}$. In Fig. 4 solid vertical lines indicate regions where the structures make a transition from RHM to LHM behavior and dashed vertical lines indicate a transition from LHM to RHM behavior.

\section{Insert Fig. 4}

In Fig. 4(a) the vertical lines indicating changes in the sign of the index are placed at $20.7 \mathrm{GHz}$ (RHM to LHM) and $22.85 \mathrm{GHz}$ (LHM to RHM). The transition from one type of behavior to the other at these points is clear because the phase lines cross so that the phase difference in equation (2) changes sign. In the region contained by these vertical lines the phase difference is positive for the two, three, and four unit cell structures, implying that the effective index is negative in this region. For the one unit cell case the phase difference is positive and the index is negative between 20.7 $\mathrm{GHz}$ and $22.95 \mathrm{GHz}$. In Fig. 4(b) the vertical lines are placed at $22.4 \mathrm{GHz}$ (RHM to LHM) and $22.65 \mathrm{GHz}$ (LHM to RHM) so that the effective index for the two, three, and four unit cell cases is negative in the region between these lines. The one unit cell case has a positive phase difference and negative index between $22.57 \mathrm{GHz}$ and $22.65 \mathrm{GHz}$. The difference in the behavior of the single and multiple unit cell cases is caused by the resonance interactions between nearest neighbor SRRs. In the one unit cell case there is a single peak because the resonance region is caused by a single SRR. In the multiple unit cell cases however, there are two peaks in the resonance region, one caused by the individual SRR resonances and the other by the interactions between nearest neighbor SRRs. This second peak in the multiple unit cell cases causes the phase plots to differ from those of the single unit cell cases.

The location of the OS transmission peak shown in Fig. 3(a) is marked in Fig. 4(a) by an arrow. A similar arrow marks the location of the SS transmission peak from Fig. 3(b) in Fig. 4(b). These arrows demonstrate a fundamental difference in the behavior of these two structures. That is, in the OS case the LHM region contains the transmission peak, whereas in the SS case the transmission peak lies outside the LHM region. Hence, in the passband, the OS structure is LHM and the SS structure is RHM. This demonstrates that the transmission magnitude does not provide the necessary information for determining the handedness of the structure. Instead the transmission phase information is required. Unfortunately since it requires transmission data for propagation through several lengths of a structure the transmission phase is not always practical as a diagnostic tool for determining the handedness of the structure. It is therefore desirable to develop an alternate diagnostic tool for this task.

\section{DISPERSION DIAGRAMS}

Using both ansoft-HFSS and FDTD software developed in house the dispersion characteristics of the SS and OS structures were simulated. The simulations were performed using the unit cells shown in Fig. 2 and periodic boundary conditions (PBC) were assigned to all faces. The phase between the $y$-faces was swept between 0 and 180 degrees. On the $x$ - and $z$-faces the phase was not varied. Figure 5 shows the simulated dispersion plots (solid lines). In each structure the light line was also simulated (dashed lines). When the mode from the light line and the mode resulting from the SRRs and SWs intersect they couple. Therefore, for comparison, using an equivalent transmission line model a mode corresponding to that of the OS structure was calculated which does not take this coupling into account (dotted line) ${ }^{2}$. In the transmission simulations of the previous section this coupling is not present because the light line mode is polarized such that its propagation is attenuated by the PEC and PMC boundaries. In the present case, the light is allowed to propagate since PBCs are used.

\footnotetext{
${ }^{1}$ All phases in Fig. 4 are plotted in such a way that their initial value $(20 \mathrm{GHz}$ in the OS case and $21 \mathrm{GHz}$ in the SS case) begins in the principal branch $\left(-180^{\circ}<\theta<180^{\circ}\right)$. The phase at higher frequencies is unwrapped starting from this point.

${ }^{2}$ This was done using a technique similar to that presented in [8].
} 


\section{Insert Fig. 5}

The band diagrams in Figs. 5(a) and 5(b) are symmetric with respect to the $\Gamma$ point (similar to any photonic crystal dispersion relation). In both Figs 5(a) and 5(b) the branches marked I correctly represent the propagation in the structures. This is because on these branches the local derivative at any point is positive (not including the light) so that the group velocity, or equally the energy velocity (we are considering propagation in the passband), is also positive [12]. In branch-I of Fig. 5(a) the slope of the line joining the origin to any point is negative, indicating that the phase velocity is negative. Therefore, this mode represents a backward wave mode (positive group but negative phase velocity), which indicates that the effective index of refraction is negative (LHM behavior) for this configuration. For branch-I in Fig. 5 (b) the group and phase velocities are both positive so that index of refraction is also positive (RHM behavior) ${ }^{3}$. At the $\Gamma$ point, it is also worth noting that the concavities (the second derivative) of the propagating modes in Figs 5(a) and 5(b) have opposite signs. These results show that in the passband the OS structure is a LHM and the SS structure is a RHM and corroborate the results from the previous section.

The aforementioned coupling between the light line and the mode created by the SRRs and SWs can also be used to confirm the LHM and RHM character of these structures. In the OS case when the light line couples with the SRR and SW mode a stop band emerges. Such behavior is characteristic of contra-directional coupling (the two interacting modes have counter propagating wave vectors). In the SS structure no stop band results when the light line couples to the SRR and SW mode, behavior which is characteristic of co-directional coupling (the two interacting modes are co-propagating).

Although it has been shown that the considered SS configuration exhibits RHM behavior in the passband it is important to note that it does exhibit some LHM behavior in the stopband. Hence, it is important to ask the following question. Can the SS structure be also made to display LHM behavior in the passband under certain conditions? We examine this in the next section by considering the effect of the unit cell size on the behavior of this structure.

\section{THE EFFECTS OF UNIT CELL SIZE}

Let us consider an array of SRRs which is isolated from any other structure that might influence its behavior. If the lattice spacing is decreased such that the resonators in the array are brought closer together the collective strength of their resonance will be enhanced. This would result in an increase of the negative value of the effective permeability. If we instead consider an isolated array of strip wires we find that the same is true. That is, reducing the lattice spacing will increase the negative value of the effective permittivity at a fixed frequency. In the composite SS (or OS) structure which includes both the SRR and SW lattices the result is the same, reducing the lattice spacing will strengthen the resonance responses of the SRRs and SWs, regardless of the fact that the structures interfere with one another. Hence in the SS structure, could it not be possible to increase the strength of the SRR and SW resonances such that they overcome the interference and exhibit LHM behavior in the passband? The band diagrams for several SS structures obtained by varying the $x$-dimension of the unit cell are shown in Fig. 6.

\section{Insert Fig. 6}

The dotted line in Fig. 6 represents the case considered in Fig. 5(b). Here, the unit cell is $4 \mathrm{~mm}$ long in the $x$ direction and the slope of the dispersion curve (the group velocity) is positive everywhere for the branch marked-I. As noted above in this case the SS structure exhibits RHM behavior in the passband and has a bandwidth of approximately $730 \mathrm{MHz}$. If the $x$-dimension is reduced from $4 \mathrm{~mm}$ to $2.5 \mathrm{~mm}$ (dashed line) the bandwidth of the passband decreases to $130 \mathrm{MHz}$. It is not clear whether this band is RHM or LHM because its slope is negative for low phase values and slightly positive at higher phase values. Further reducing the unit cell size to $1.5 \mathrm{~mm}$ in the $x$-direction results in an LHM band with a bandwidth of $250 \mathrm{MHz}$, the behavior of which is described by branch-II of Fig. 6 (solid line). Hence,

\footnotetext{
${ }^{3}$ This is not to say that a negative group velocity (branch II) is unphysical. Such behavior has been observed but only in the anomalous dispersion region when passive media are concerned. Since we are considering propagation in the passband of a passive medium the case of negative group velocity does not apply here.
} 
by reducing the lattice spacing and increasing the resonance response of the SRRs and SWs the SS made a transition from RHM to LHM behavior, with the transition unit cell size being approximately $2.5 \mathrm{~mm}$ where the passband was nearly flat. Once again the coupling between the light line and the SRR and SW mode can be used to confirm the sign of the index obtained from the dispersion diagrams. As the unit cell size was decreased the coupling made a transition from co-directional coupling (RHM-RHM) in the 4mm case to contra-directional coupling (RHM - LHM) in the $1.5 \mathrm{~mm}$ case.

From the above analysis it is clear that both the OS and SS configurations of the SRRs and SWs can be used to produce LHM behavior. However, because of the interference between the two components in the SS case the lattice spacing must be smaller than that of the OS case to obtain this behavior. Therefore the interference does not completely destroy the potential for LHM behavior, it merely weakens it.

\section{CONCLUSIONS}

The behavior of two composite SRR and SW structures was examined. In the first structure, the rings and strips were printed on opposite sides of the substrate (OS structure), and in the second structure, the rings and strips were printed on the same side of the substrate (SS structure). Although both structures exhibited a transmission peak in the region where the real parts of the electric permittivity and magnetic permeability were expected to be negative it was shown that this is not sufficient evidence to determine whether the structure is RHM or LHM. To determine the handeness of the structure other diagnostic tools such as the insertion phase for propagation through several lengths of the structure or the dispersion diagrams are necessary. The effect of the unit cell size on the behavior of the SS structure was also investigated and it was shown that by modifying the unit cell dimensions the structure could be made either RHM or LHM.

\section{REFERENCES}

[1] V. G. Veselago, "The electrodynamics of substances with simultaneous negative values of $\varepsilon$ and $\mu$ “, Sov. Phys. Usp. 10, 509, 1968.

[2] J. B. Pendry, A. J. Holden, W. J. Stewart, I. Youngs, "Extremely low frequency plasmons in metallic mesostructures", Phys. Rev. Lett 76, 4773, 1996.

[3] J. B. Pendry, A. J. Holden, D. J. Robbins, and W. J. Stewart, "Magnetism from conductors and enhanced nonlinear phenomena", IEEE Trans. on Microwave Theory and Techniques 47, 2075, 1999.

[4] D. R. Smith, W. J. Padilla, D. C. Vier, S. C. Nemat-Nasser, and S. Schultz, "Composite medium with simultaneous negative permeability and permittivity", Phys. Rev. Lett. 84, 4184, 2000.

[5] R. A. Shelby, D. R. Smith, S. Schultz, "Experimental verification of a negative index of refraction", Science 292, 77, 2001.

[6] G. V. Eleftheriades, A. K. Iyer, P. C. Kremer, "Planar negative refractive index media using periodically $L-C$ loaded transmission lines", IEEE Trans. On Microwave Theory and Techniques 50, 2702, 2002.

[7] M. Bayindir, K. Aydin, E. Ozbay, P. Markos, C. M. Soukoulis, "Transmission properties of composite metamaterials in free space", Appl. Phys. Lett., 81, 2002.

[8] O. F. Siddiqui, M. Mojahedi, G. V. Eleftheriades, "Periodically loaded transmission line with effective negative refractive index and negative group velocity", IEEE Trans. On Antennas and Propagation 51, 2619, 2003.

[9] O. F. Siddiqui, S. J. Erickson, G. V. Eleftheriades, "Time-domain measurement of negative group delay in negative-refractive-index transmission-line metamaterials", IEEE Trans. on Microwave Theory and Techniques 52, 1449, 2004.

[10] C. Caloz, H. Okabe, H. Iwai, and T. Itoh, "Application of the transmission line theory of left-handed (LH) metamaterials to the realization of a microstrip "LH line" ", IEEE AP-S/URSI Internatianal Symp., San Antonio, TX, 2002.

[11] R. W. Ziolkowsky, "Design, fabrication, and testing of double negative metamaterials", IEEE Trans. on Antennas and Propagation 51, 1516, 2003.

[12] J. F. Woodley, M. Mojahedi, "Negative group velocity and group delay in left-handed media", Phys. Rev. E 70, 2004.

[13] R. A. Shelby, D. R. Smith, S. C. Nemat-Nasser, and S. Schultz, "Microwave transmission through a twodimensional, isotropic, left-handed metamaterial", App. Phys. Lett., 78, 489, 2001. 


\section{Figure Captions}

FIG. 1. Schematic of the split ring resonator. The dimensions used in the simulations were $r=0.506 \mathrm{~mm}, c=0.124 \mathrm{~mm}, d=0.15$ $\mathrm{mm}$, and $g=0.114 \mathrm{~mm}$.

FIG. 2. (a) Opposite side (OS) structure. (b) Same side (SS) structure. In each case the metallic strip was $0.5 \mathrm{~mm}$ wide and the substrate was $0.5 \mathrm{~mm}$ thick with a dielectric constant of 3.02. The dimensions of the unit cell in the OS configuration were $2.5 \times 2.5 \times 2.5 \mathrm{~mm}$. In the SS configuration the dimensions were $4 \times 2.5 \times 2.5 \mathrm{~mm}$.

FIG. 3. Simulated transmission magnitude through 4 unit cell long (a) OS structure, and (b) the SS structure. Solid (dashed) vertical lines indicate the points at which the structures make transitions from RHM (LHM) to LHM (RHM) behavior.

FIG. 4. (Color online) Transmission phase for propagation through 1, 2, 3, and 4 unit cells of a) OS structure and (b) SS structure. Solid (dotted) vertical lines indicate the points at which the structures make transitions from RHM (LHM) to LHM (RHM) behavior. Arrows mark the locations of the transmission peaks from the corresponding OS or SS plots in Fig. 3.

FIG. 5. (Color online) Dispersion plots for (a) OS structure and (b) SS structure. In the OS configuration the unit cell size was $2.5 \times 2.5 \times 2.5 \mathrm{~mm}$. The unit cell size was $4 \times 2.5 \times 2.5 \mathrm{~mm}$ in the SS case.

FIG. 6. (Color online) Dispersion curves for the SS structure. In each case the $y$-and $z$-dimensions of the unit cell were $2.5 \mathrm{~mm}$ and the $x$-dimension was varied. 


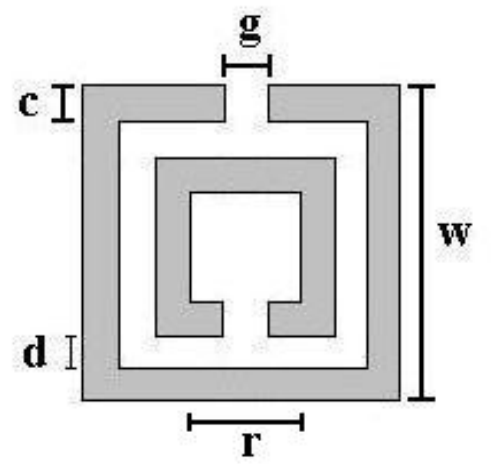

FIG. 1.
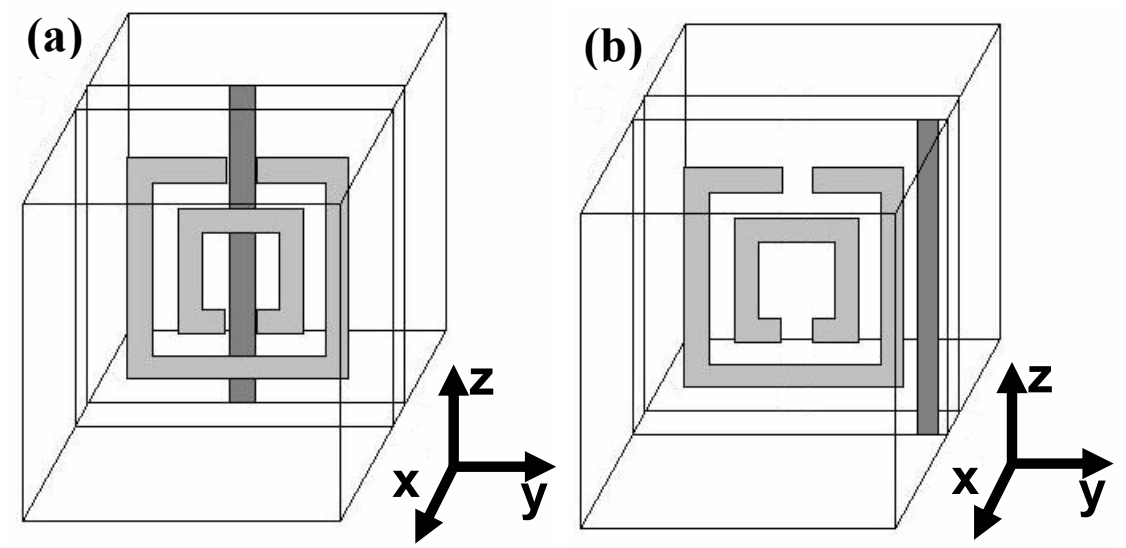

FIG. 2. 

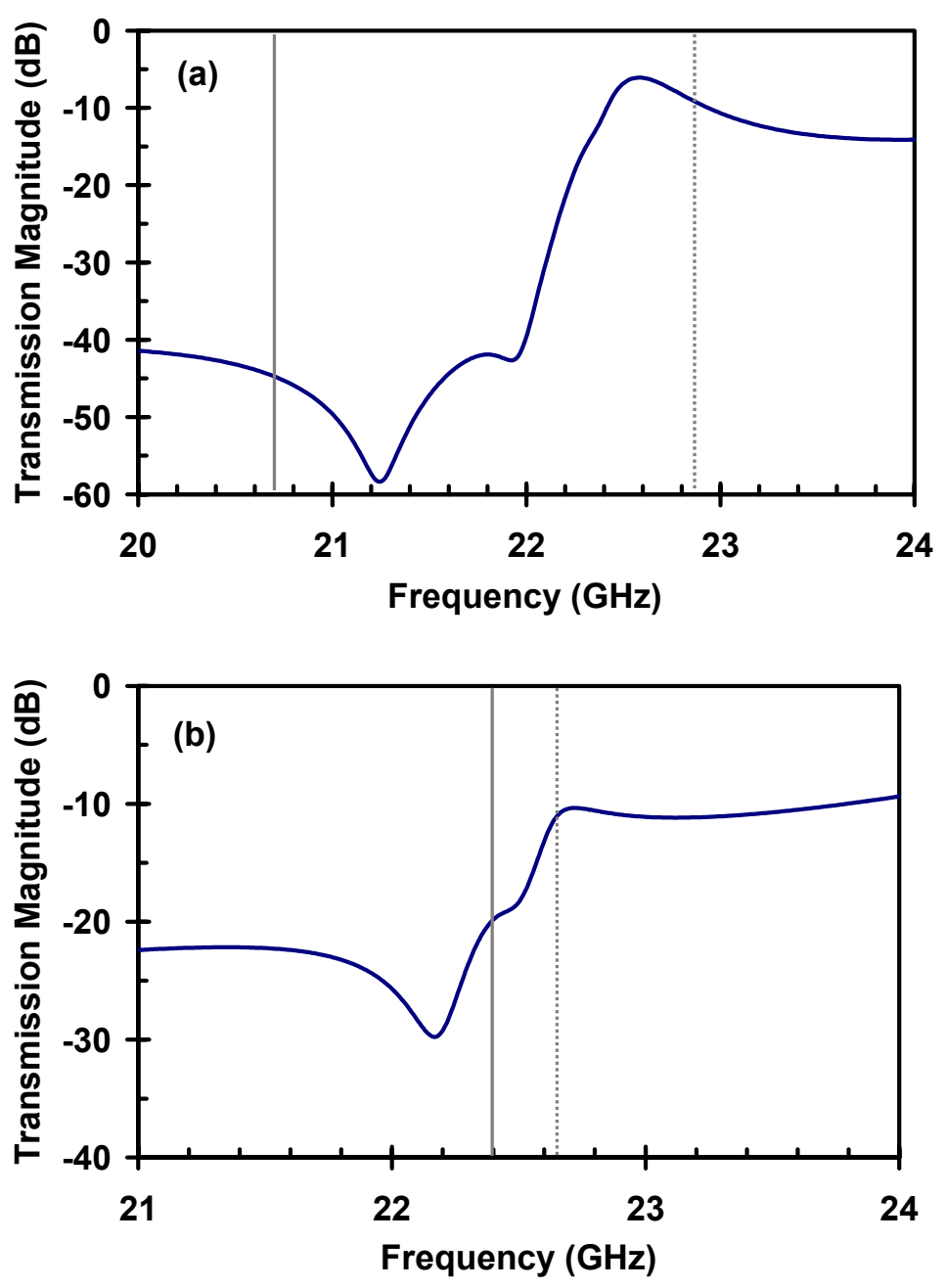

FIG. 3. 

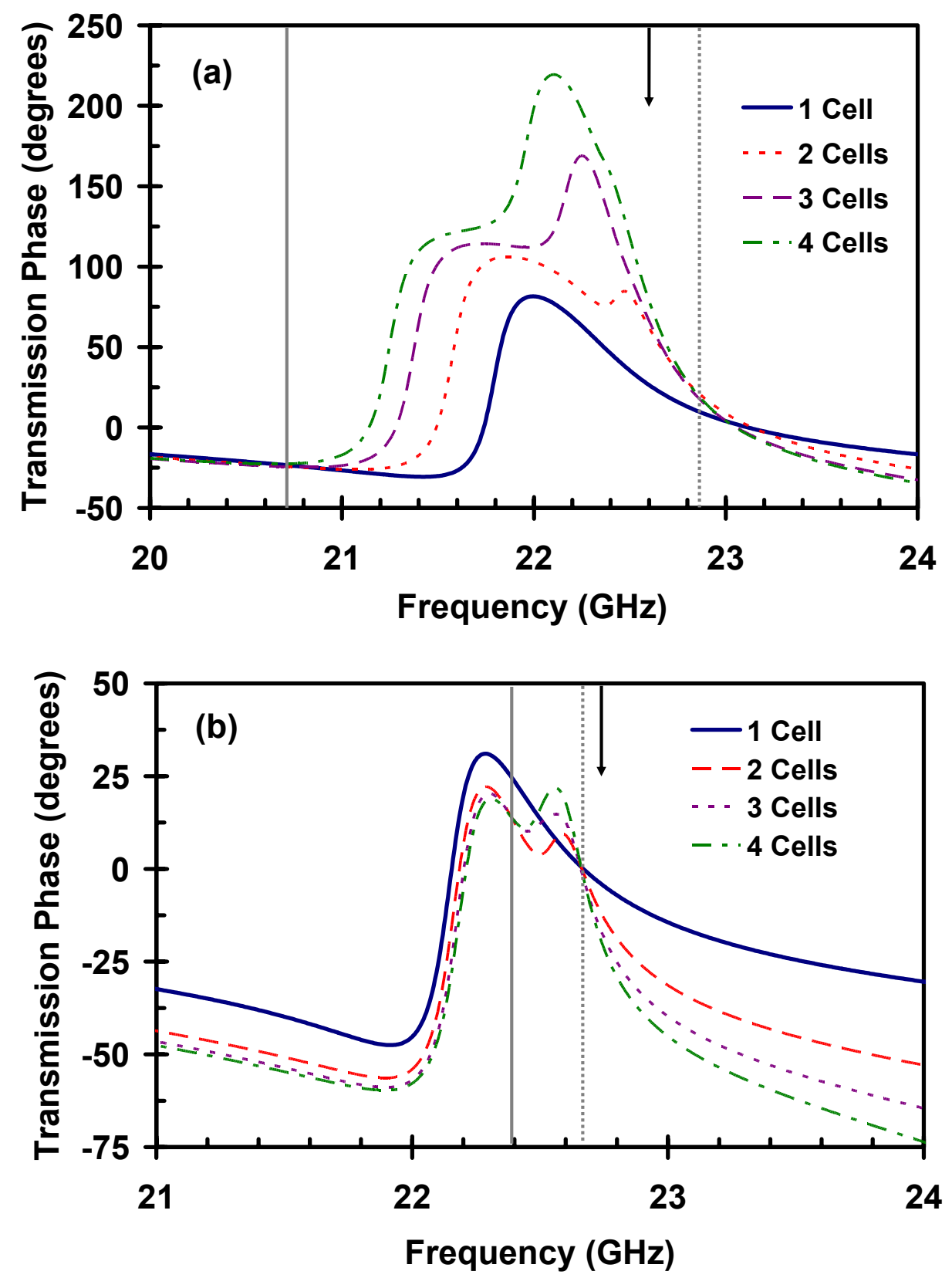

FIG. 4 . 

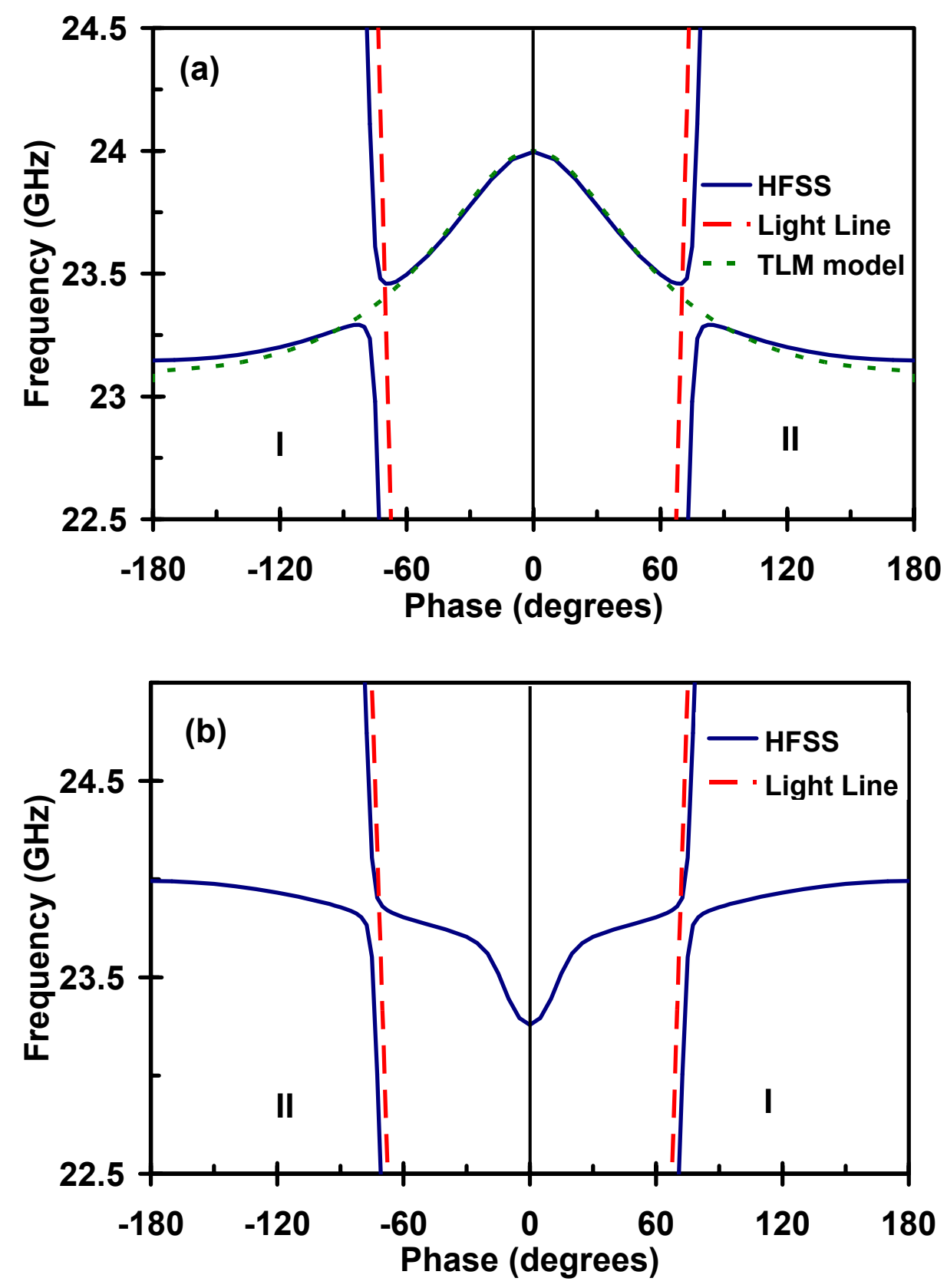

FIG. 5. 


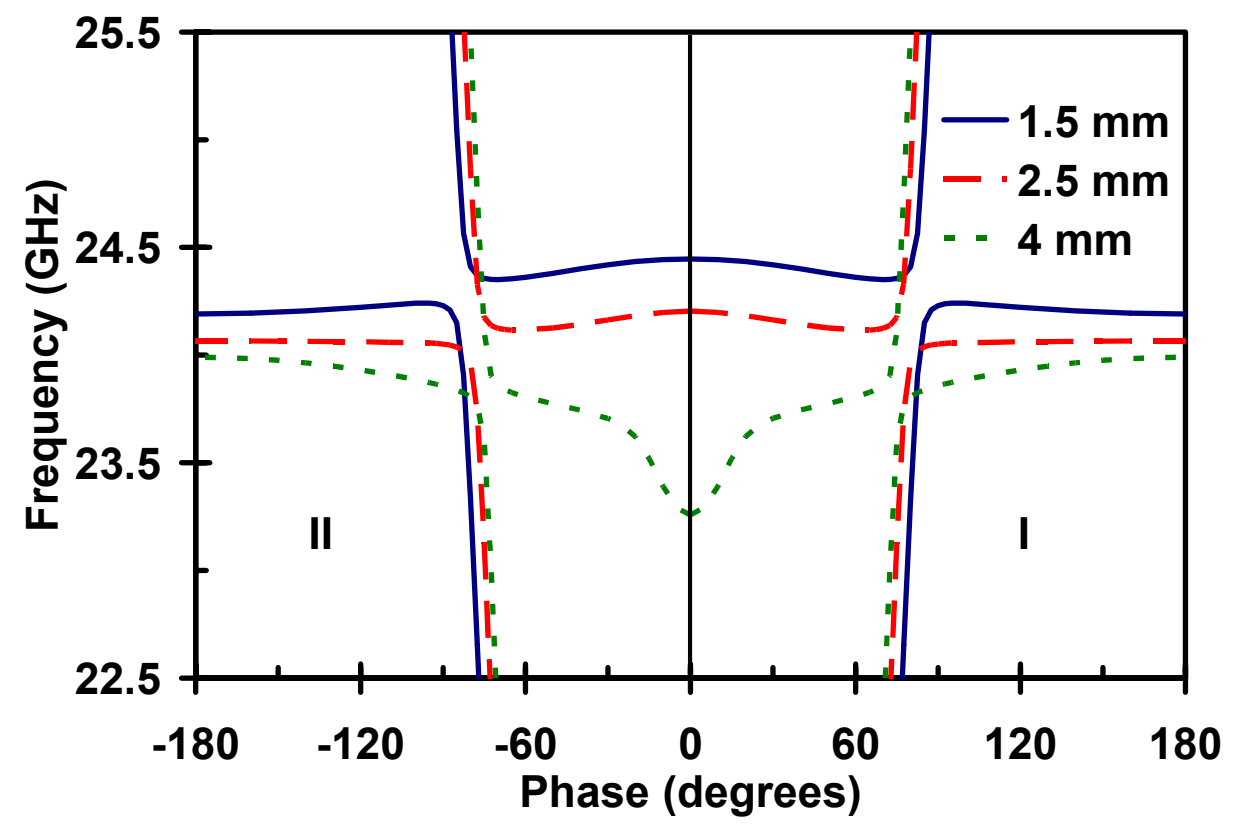

FIG. 6. 